\title{
Structural Studies of Nonclassical Cyclobutylmethyl Cations by the ab initio Method
}

\author{
V. Prakash Reddy, 'Golam Rasul, G. K. Surya Prakash and George A. Olah" \\ Department of Chemistry, University of Missouri-Rolla, Rolla, MO 65409; email:

$$
\text { preddy@umr.edu }
$$ \\ Loker Hydrocarbon Research Institute and Department of Chemistry, University of \\ Southern California, Los Angeles, CA 90089; email: olah@usc.edu
}

\section{Table of Contents}

Cartesian coordinates for $\mathbf{8}$

Cartesian coordinates for 9

Cartesian coordinates for $\mathbf{1 0}$

Cartesian coordinates for $\mathbf{1 1}$

Cartesian coordinates for $\mathbf{1 2}$

S3

Cartesian coordinates for $\mathbf{1 3}$

S4

Reference $\mathbf{3 3}$

S4 
Cartesian coordinates of the MP2/cc-pVTZ optimized geometries of 8 -10 and $\mathbf{1 1}-\mathbf{1 3}$.

$\begin{array}{lrcc}\text { 8 } & & & \\ \text { C } & 0.7447 & -1.0072 & -0.1967 \\ \text { C } & -0.7450 & -1.0070 & 0.1967 \\ \text { C } & 0.0001 & 1.2478 & 0.0000 \\ \text { C } & -1.1873 & 0.4428 & -0.0908 \\ \text { C } & 1.1874 & 0.4426 & 0.0909 \\ \text { H } & 2.0858 & 0.8513 & -0.3747 \\ \text { H } & 1.3819 & 0.5684 & 1.1895 \\ \text { H } & 0.8719 & -1.2099 & -1.2610 \\ \text { H } & 0.0003 & 2.3350 & -0.0002 \\ \text { H } & -1.3819 & 0.5691 & -1.1894 \\ \text { H } & -2.0856 & 0.8518 & 0.3748 \\ \text { H } & -0.8722 & -1.2098 & 1.2609 \\ \text { H } & 1.3347 & -1.7353 & 0.3515 \\ \text { H } & -1.3352 & -1.7350 & -0.3516 \\ & & & \\ \text { 9 } & & & \\ \text { C } & 0.7447 & -1.0072 & -0.1967 \\ \text { C } & -0.7450 & -1.0070 & 0.1967 \\ \text { C } & 0.0001 & 1.2478 & 0.0000 \\ \text { C } & -1.1873 & 0.4428 & -0.0908 \\ \text { C } & 1.1874 & 0.4426 & 0.0909 \\ \text { H } & 2.0858 & 0.8513 & -0.3747 \\ \text { H } & 1.3819 & 0.5684 & 1.1895 \\ \text { H } & 0.8719 & -1.2099 & -1.2610 \\ \text { H } & 0.0003 & 2.3350 & -0.0002 \\ \text { H } & -1.3819 & 0.5691 & -1.1894 \\ \text { H } & -2.0856 & 0.8518 & 0.3748 \\ \text { H } & -0.8722 & -1.2098 & 1.2609 \\ \text { H } & 1.3347 & -1.7353 & 0.3515 \\ \text { H } & -1.3352 & -1.7350 & -0.3516 \\ & & & \\ \mathbf{1 0} & & & \\ \text { C } & -0.4189 & 1.0280 & -0.3970 \\ \text { C } & 0.9767 & 0.7801 & 0.1937 \\ \text { C } & -0.3749 & -1.0610 & -0.1881 \\ \text { C } & 1.0575 & -0.7518 & 0.0295 \\ \text { C } & -1.2926 & -0.1553 & 0.3750 \\ \text { H } & -2.3327 & -0.2492 & 0.0955 \\ \text { H } & -1.1188 & 0.1264 & 1.4101 \\ \text { H } & -0.4357 & 0.9280 & -1.4820 \\ \text { H } & -0.6986 & -1.7917 & -0.9262\end{array}$




$\begin{array}{rrrr}\mathrm{H} & 1.6386 & -1.0598 & -0.8376 \\ \mathrm{H} & 1.4464 & -1.3109 & 0.8871 \\ \mathrm{H} & 1.0103 & 1.0840 & 1.2370 \\ \mathrm{H} & -0.9589 & 1.9318 & -0.1201 \\ \mathrm{H} & 1.7627 & 1.3016 & -0.3423\end{array}$

11

$\begin{array}{llll}\text { C } & -0.4879 & 1.1852 & 0.0709\end{array}$

$\begin{array}{llll}\text { C } & -1.4186 & 0.2523 & -0.7429\end{array}$

$\begin{array}{llll}\text { C } & -0.1267 & -0.3534 & 0.9686\end{array}$

$\begin{array}{llll}\text { C } & -1.0915 & -1.0543 & 0.0236\end{array}$

$\begin{array}{llll}\text { C } & 1.1128 & 0.1347 & 0.5749\end{array}$

$\begin{array}{llll}\text { C } & 1.8933 & -0.2497 & -0.6354\end{array}$

$\begin{array}{llll}\mathrm{H} & 1.6297 & 0.7545 & 1.3048\end{array}$

$\begin{array}{llll}\mathrm{H} & -0.9260 & 1.7934 & 0.8581\end{array}$

$\begin{array}{llll}\mathrm{H} & -0.4180 & -0.2002 & 2.0036\end{array}$

$\begin{array}{llll}\mathrm{H} & -1.9390 & -1.4805 & 0.5555\end{array}$

H $-0.6331 \quad-1.8393-0.5770$

$\mathrm{H}-1.1614 \quad 0.2274 \quad-1.8005$

$\begin{array}{lllll}\mathrm{H} & 0.2265 & 1.7908 & -0.4858\end{array}$

$\begin{array}{llll}\mathrm{H} & -2.4582 & 0.5647 & -0.6493\end{array}$

H $1.3037 \quad-0.7398 \quad-1.4082$

H $2.6583 \quad-0.9609 \quad-0.2984$

$\begin{array}{lllll}\mathrm{H} & 2.4291 & 0.6007 & -1.0603\end{array}$

12

$\begin{array}{llll}\text { C } & -0.2927 & 1.0477 & 0.3373\end{array}$

$\begin{array}{llll}\text { C } & -1.6728 & 0.6332 & -0.2084\end{array}$

$\begin{array}{llll}\text { C } & -0.0291 & -0.8052 & 0.3649\end{array}$

$\begin{array}{llll}\text { C } & -1.4801 & -0.8996 & -0.0749\end{array}$

$\begin{array}{llll}\text { C } & 0.9248 & -0.1801 & -0.4256\end{array}$

$\begin{array}{llll}\text { C } & 2.3526 & 0.0076 & -0.0214\end{array}$

H $\quad 0.6872 \quad-0.0192 \quad-1.4755$

$\begin{array}{llll}\mathrm{H} & -0.2088 & 1.1993 & 1.4125\end{array}$

$\begin{array}{llll}\mathrm{H} & 0.2683 & -1.1377 & 1.3573\end{array}$

H $-2.1006-1.3506 \quad 0.6969$

H $-1.6121 \quad-1.4659-0.9979$

$\mathrm{H}-1.8179 \quad 0.9616 \quad-1.2370$

H $\quad 0.2576 \quad 1.8440 \quad-0.1650$

$\begin{array}{llll}\mathrm{H} & -2.4861 & 1.0353 & 0.3947\end{array}$

H $2.4986 \quad-0.0685 \quad 1.0571$

$\begin{array}{llll}\mathrm{H} & 2.7514 & 0.9599 & -0.3755\end{array}$

H $2.9466 \quad-0.7791 \quad-0.4989$ 


$\begin{array}{lrrr}13 & & & \\ \text { C } & -0.9433 & -0.9011 & 0.7278 \\ \text { C } & -2.2200 & -0.1392 & 0.3065 \\ \text { C } & -0.2332 & -0.2199 & -0.6365 \\ \text { C } & -1.4320 & 0.7447 & -0.6945 \\ \text { C } & 1.0683 & 0.0711 & -0.1391 \\ \text { C } & 1.4042 & 1.3652 & 0.4915 \\ \text { C } & 2.1248 & -0.9593 & -0.2188 \\ \text { H } & -0.9195 & -1.9874 & 0.6822 \\ \text { H } & -0.2443 & -0.9996 & -1.3976 \\ \text { H } & -1.8646 & 0.8442 & -1.6891 \\ \text { H } & -1.2161 & 1.7426 & -0.3132 \\ \text { H } & -2.7277 & 0.3800 & 1.1183 \\ \text { H } & -0.5068 & -0.5575 & 1.6649 \\ \text { H } & -2.9300 & -0.7997 & -0.1915 \\ \text { H } & 1.8023 & -1.8890 & -0.6842 \\ \text { H } & 2.5570 & -1.1561 & 0.7688 \\ \text { H } & 2.9548 & -0.5397 & -0.8072 \\ \text { H } & 1.6215 & 2.0644 & -0.3331 \\ \text { H } & 2.2899 & 1.3178 & 1.1237 \\ \text { H } & 0.5709 & 1.8108 & 1.0361\end{array}$

\section{Reference 33:}

Gaussian 03 (Revision B.04), F., M. J.; Trucks, G. W.; Schlegel, H. B.; Scuseria, G. E.; Robb, M. A.; Cheeseman, J. R.; Montgomery, J. A.; Vreven, T.; Kudin, K. N.; Burant, J. C.; Millam, J. M.; Iyengar, S. S.; Tomasi, J.; Barone, V.; Mennucci, B.; Cossi, M.; Scalmani, G.; Rega, N; Petersson, G. A.; Nakatsuji, H.; Hada, M.; Ehara, M.; Toyota, K.; Fukuda, R.; Hasegawa, J.; Ishida, M.; Nakajima, T.; Hona, Y.; Kitao, K.; Nakai, H.; Klene, M.; Li, X.; Knox, J. E.; Hratchian, H. P.; Cross, J. B.; Adamo, C.; Jaramillo, J.; Gomperts, R.; Stratmann, R. E.; Yazyev, O.; Austin, A. J.; Cammi, R.; Pomelli, C.; Ochterski, J. W.; Ayala, P. Y.; Morokuma, K.; Voth, G. A.; Salvador, P.; Dannenberg, J. J.; Zakrzewski, V. G.; Dapprich, S.; Daniels, A. D.; Strain, M. C.; Farkas, O.; Malick, D. K.; Rabuck, A. D.; Raghavachari, K.; Foresman, J.; Ortiz, J. V.; Cui, Q.; Baboul, A. G.; Clifford, S.; Cioslowski, J.; Stefanov, B. B.; Liu, G.; Liashenko, A.; Piskorz, P.; Komaromi, I.; Martin, R. L.; Fox, D. J.; Keith, T.; Al-Laham, M. A.; Peng, C. Y.; Nanayakkara, A.; Challacombe, M.; Gill, P. M. W.; Johnson, B.; Chen, W.; Wong, M. W.; Gonzalez, C.; Pople, J. A.,Gaussian, Inc., Pittsburgh PA, 2003. 